


\title{
RE-17 \\ OPTIMIZATION OF MIDCOURSE VELOCITY CORRECTIONS
}

by

\author{
Robert G. Stern \\ and \\ James E. Potter
}

Note: This report was presented at the symposium on Automatic Control in the Peaceful Uses of Space, sponsored by the International Federation of Auto-

matic Control, on 21 June 1965 in Stavanger, Norway.

\section{EXPERIMENTAL ASTRONOMY LABORATORY MASSACHUSETTS INSTITUTE OF TECHNOLOGY CAMBRIDGE 39, MASSACHUSETTS}


TABLE OF CONTENTS

Page

1. INTRODUCTION. ................. 1

2. MIDCOURSE GUIDANCE THEORY........... 2

3. CRITICAL-PLANE COORDINATE SYSTEM. . . . . . . . 4

4. SINGLE-CORRECTION STRATEGY .......... 6

5. MULTIPLE-CORRECTION STRATEGIES . . . . . . . 8

6. MULTIPLE-CORRECTION STRATEGY FOR POSITION-CONSTRAINED VTA GUIDANCE . . . . . . 10

REFERENCES ...................... 14 


\title{
OPTIMIZATION OF MIDCOURSE VELOCITY CORRECTIONS
}

\author{
by Dr. Robert G. Stern and Dr. James E. Potter \\ Staff Engineers Experimental Astronomy Laboratory \\ Massachusetts Institute of Technology \\ Cambridge, Massachusetts
}

\section{ABSTRACT}

The concept of a six-dimensional state space is used to develop the fundamental equations of linearized midcourse guidance. Both fixed endpoint (fixed-time-of-arrival) and variable endpoint (variable-time-of-arrival) problems are considered. It is shown that the variable-timeof-arrival problem can be simplified mathematically by the introduction of a special coordinate system, which is called the critical-plane coordinate system.

A method is developed for determining the optimum time at which to apply a single midcourse correction the effect of which is to satisfy a set of position constraints. The correction is "optimum" in the sense that its magnitude is minimized. For a given nominal trajectory, the time of the correction depends on the predicted miss vector at the destination. The method is particularly simple to apply in the case of variable-time-of-arrival guidance; by exploiting the critical-plane coordinate system, a single curve can be prepared prior to the flight to indicate the optimum correction time as a function of a miss parameter which is determined from in-flight navigational measurements.

Multiple-correction strategies are then investigated. A method is developed for determining an optimum schedule of midcourse corrections. The optimum schedule is the one for which the sum of the magnitudes of all corrections is minimized. It is proved that the number of corrections in an optimum schedule is no greater than the number of constraints to be satisfied at the nominal time of arrival at the destination.

In position-constrained variable-time-ofarrival guidance there are only two constraints at the nominal time of arrival; hence there are at most two corrections in the optimum schedule. The optimum two-correction strategy is compared with the optimum single-correction strategy. It is shown that for certain ranges of the miss parameter two corrections can effect a saving in total magnitude of velocity correction, while in other ranges no improvement can be obtained from two corrections. A geometric construction, based on the theory of convex sets, is used to determine the ranges of miss parameter in which two corrections a re preferable, and also the times and components of both corrections when they are preferable.

It may be noted that the developments in this paper are deterministic rather than statistical. No consideration is given to the uncertainties of the navigational measurements; it is assumed that a sufficient number of measurements has been made during the flight so that the error in the predicted miss vector at the destination is negligible. The control action taken is determined by the predicted miss vector.

\section{INT RODUCTION}

Midcourse guidance systems for space vehicles can be separated into two classes - fixed-time-ofarrival (FTA) systems and variable-time-ofarrival (VTA) systems. In the former a set of constraints on the vehicle's state relative to the target body must be satisfied at a specified terminal time; in the latter the same types of constraints must be satisfied, but the actual terminal time at which they are satisfied is permitted to vary slightly from the predetermined nominal terminal time. By relaxing the specification on time of arrival, the VTA system permits more flexibility in the development of guidance laws.

The constraints most commonly specified are the three components of the vehicle's position relative to the target. Battin(1); Noton, Cutting, and Barnes(2), and McLean, Schmidt, and McGee(3), among others, have shown how linear theory can be applied to compute the components of a single small step change in velocity that will satisfy a set of three position constraints. The theory depends on the availability of a precomputed reference trajectory relative to which the vehicle's actual trajectory can be defined. The components of the required velocity change, usually referred to as the "correction, "vary linearly with the predicted deviation of the vehicle's actual position from the desired position at the nominal time of arrival at the destination.

Breakwell(4), (5) attacks the problem of selecting the optimum times at which a series of midcourse corrections should be made. The optimization criterion is that the total fuel expenditure (i. e., the sum of the magnitudes of the velocity steps) be minimized. The analysis is statistical, based on a priori knowledge of the variances of the uncertainties in the vehicle's initial state, in the observations, and in the corrections applied. A more recent statistical study, containing computer results for a number of simulated interplanetary missions, has been made by White, Callas, and Cicolani. (6)

The present paper, like those just cited, deals with optimizing a correction schedule. First, a method is developed for determining the optimum

\footnotetext{
"Superior numbers refer to similarly-numbered references at the end of this paper.
} 
time at which to apply a single midcourse correction that satisfies all the position constraints. Then optimum multiple-correction strategies are investigated and compared with the optimum single-correction strategy. The strategies developed in this paper, unlike those in the previous papers, are deterministic rather than statistical. The correction schedule depends on the predicted position variation at the nominal time of arrival at the destination; it does not depend on the uncertainties in the measured or controlled quantities.

As a preliminary to the development of the optimization procedure with which this paper is primarily concerned, the next two sections formulate the basic equations of midcourse guidance and describe the critical-plane coordinate system, which simplifies the analysis of VTA guidance.

\section{MIDCOURSE GUIDANCE THEORY}

The position and velocity of a space vehicle on its trajectory can be represented by the sixcomponent state vector $x$.

$$
\underline{x}=\left[\begin{array}{l}
\underline{r} \\
\underline{v}
\end{array}\right]
$$

$r$ and $\mathrm{v}$ are the position and velocity vectors, respectively, of the vehicle with respect to the origin of the coordinate system. The equations of motion of the vehicle have the form

$$
\underline{\dot{x}}=\underline{f}(\underline{x}, t)+\stackrel{\dot{*}}{M} \sum_{k=1}^{p} \stackrel{\mathrm{c}}{\mathrm{c}} \delta\left(t-t_{k}\right)
$$

f the rate of change of the state rector with $\bar{t}_{\mathrm{me}}$ in the absenee of any control action. $\mathrm{c}$ is a corrective velocity change resulting from an acceleration impulse applierl at time $t_{k}$. $i\left(t-t_{k}\right)$ is the IDrae delta function at $t_{k}$. There are $p$ impulses cluring the flight. $M^{*}$ is a $6-b y-3$ compatibility matrix, relating the six-dimensional $\underline{x}$ to the three-dimensional $\mathrm{c} k$

$$
\stackrel{*}{M}=\left[\begin{array}{l}
* \\
0_{3} \\
* \\
\mathrm{I}_{3}
\end{array}\right]
$$

$\hat{0}_{3}$ and $\overrightarrow{\mathrm{I}}_{3}$ are the $3-\mathrm{by}-3$ zero matrix and the 3 -by -3 identity matrix, respectively.

The solution of Eq. (2.2) with ideal initial conditions and no corrective impulses constitutes the nominal, or reference, trajectory. This solution is precomputed numerically and stored for use during the flight. Because the actual initial conditions are not ideal, inflight corrections are required; the corrective acceleration at time $t_{k}$ produces a step change $c k$ in the velocity at $t_{k}$. To first order in the variation $\delta \underline{x}_{I}$ of the initial state from its nominal value and to first order in the velocity corrections $c k$, the variation $\delta x \mathrm{D}$ in the state vector at the nominal time of arrival at the destination is given by

$$
\begin{aligned}
& \delta \underline{x}_{D}=\psi_{D I}^{*} \underline{x}_{I}+\sum_{k=1}^{p} \stackrel{*}{\Phi}_{D k}^{* *} \underline{M}_{-k} \\
& =\delta \underline{x}_{D}-\sum_{k=1}^{p} \stackrel{\kappa}{\Phi}_{D k} \stackrel{*}{M}_{-k}
\end{aligned}
$$

$\delta \mathrm{x}^{-}{ }^{-}$is the state vector variation that would exist at the nominal time of arrival if no corrections were applied. $\Phi_{j i}$ is the $6-b y-6$ state transition
matrix.

$$
\stackrel{*}{\Phi i}_{j i}=\left[\frac{\partial \underline{x}_{j}}{\partial \underline{x}_{i}}\right]
$$

For fixed $t_{i}$ and variable $t_{j}$ the elements of $\stackrel{*}{\Phi}_{j i}$ can be determined by numerical integration, as shown in Ref. (1).* It is convenient for analytic work to partition $\boldsymbol{\Phi}_{j i}$ into four 3 -by-3 submatrices.

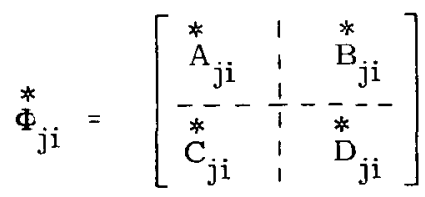

Let the number of constraints to be satisfied at the destination be $m$. In general, the constraints are functions of the position and velocity (i. e., of the state) of the vehicle relative to the target at the actual time of arrival. In FTA guidance the actual arrival time $t_{A}$ is the same as the nominal arrival time $t_{D}$; in VTA guidance the two arrival times are usually not the same. The relative state of the vehicle at $t_{A}$ is designated $x$ RA.

$$
\underline{x}_{\mathrm{RA}}=\underline{\mathrm{x}}_{\mathrm{A}}-\underline{\mathrm{x}}_{\mathrm{TA}}
$$

$x A$ and $x T A$ are, respectively, the state of the vehicle and the state of the target at $t A$. The constraint equations may be written in the form

$$
\gamma_{k}\left(x_{R A}\right)=0 ; k=1, \ldots . m
$$

Since $x$ RA is a six-component vector, the maximum number of linearly independent constraints is six. The vector form of (2.8) is

$$
\underline{\gamma}=\underline{0} \mathrm{~m}
$$

where $0 \mathrm{~m}$ is the $\mathrm{m}$-component zero vector.

If the vehicle is on the reference trajectory, all the constraints are satisfied at $t_{D}$. On the actual trajectory, satisfying a particular constraint $\gamma_{k}$ requires thit, to first order,

$$
\left.\delta \gamma_{\mathrm{k}}=\underline{\mathrm{h}}_{\mathrm{k}}^{\mathrm{T}} \underline{\underline{x}}_{\mathrm{RA}}-\underline{\mathrm{x}}_{\mathrm{RD}}\right)=0
$$

where $h_{k}$ is a six-component column vector of partial derivatives. 


$$
\underline{\mathrm{h}}_{\mathrm{k}}=\left[\begin{array}{l|l}
\frac{\partial \gamma_{\mathrm{k}}}{\partial \underline{\mathrm{x}}} & \mathrm{t}=\mathrm{t}_{\mathrm{D}}
\end{array}\right]
$$

$\mathrm{x} R \mathrm{R}$ is the relative state at $t_{\mathrm{D}}$. Note that $\delta \underline{\mathrm{x}} \mathrm{D}$ is the state variation of the actual trajectory from the reference trajectory at the nominal time of arrival, while ( $x R A-x R D$ ) is the difference between the relative state of the actual trajectory at the actual time of arrival and the relative state of the reference trajectory at the nominal time of arrival. The difference in relative states can be determined as follows:

$$
\begin{aligned}
\underline{x}_{R A}= & \left(\underline{x}_{D}+\delta \underline{x}_{D}+\underline{f}_{D} \delta t_{D}\right) \\
& -\left(\underline{x}_{T D}+\underline{f}_{T D} \delta t_{D}\right) \\
= & \underline{x}_{R D}+\underline{x}_{D}+\underline{f}_{R D} \delta t_{D} \\
\underline{x}_{R A}- & \underline{x}_{R D}=\delta \underline{x}_{D}+\underline{f}_{R D} \delta t_{D}
\end{aligned}
$$

$\delta t_{D}$ is the change in time of arrival. $f R D$ is the time rate of change of the relative state at $t_{D}$.

$$
\begin{gathered}
\delta t_{D}=t_{A}-t_{D} \\
\underline{f}_{R D}=\underline{f}_{D}-\underline{f}_{T D}
\end{gathered}
$$

The variational form of the $i-$ th constraint equation can now be written as

$$
\begin{aligned}
\delta \gamma_{i}= & 0=\underline{h}_{i}^{T}\left(\delta \underline{x}+\underline{f}_{R D} \delta t_{D}\right) \\
= & \underline{h}_{i}^{T}\left[\delta \underline{x}_{D}^{-}+\sum_{k=1}^{p}{ }^{*}{ }_{D k}^{*}{ }^{*} \underline{c}_{k}\right. \\
& \left.+\underline{f}_{R D} \delta t_{D}\right]
\end{aligned}
$$

For FTA guidance $\delta t$ is equal to zero. All m constraints can be combined into a single vector equation.

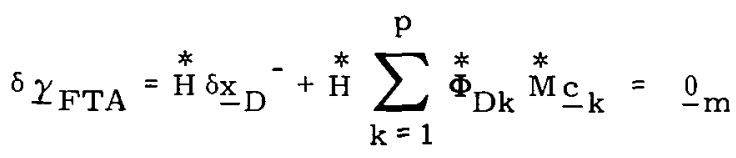

where the $\mathrm{m}$-by- 6 matrix $\mathrm{H}^{*}$ is given by

$$
\text { * }=\left[\begin{array}{c}
\underline{\mathrm{h}}_{1}^{\mathrm{T}} \\
\dot{\mathrm{h}^{2}} \\
\underline{\mathrm{m}}_{\mathrm{m}}^{\mathrm{T}}
\end{array}\right]
$$

In VTA guidance one of the constrants can be used as a "stopping" condition, that is, as a means of determining $\delta t_{D}$. This is true because $\delta t_{D}$ is not an independent va riable but is actually dependent on $\delta \times \mathrm{D}$. The constraint used for this determination must be one for which the scalar product of ${ }_{\mathrm{h}} \mathrm{k}$ and $\mathrm{f} \mathrm{RD}$ is not equal to zero. Suppose that

$\bar{\gamma}_{\mathrm{m}}$ meets this requirement. Then, from $(2.16)$,

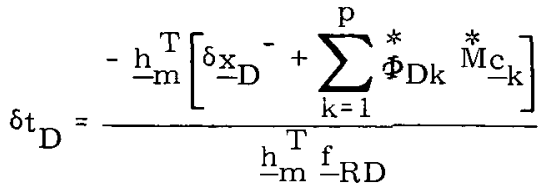

The variational equation of the $i$-th constraint (where $\mathbf{i} \neq \mathrm{m}$ ) becomes

$$
\begin{aligned}
& \delta \gamma_{i}=\underline{h}_{\mathbf{i}}^{\mathrm{T}}\left[\ddot{H}_{6}-\frac{\underline{\mathrm{f}} \mathrm{RD} \frac{\mathrm{h}}{\mathrm{I}^{\mathrm{T}}}}{\underline{\mathrm{h}}_{\mathrm{m}}^{\mathrm{T}} \underline{\mathrm{f}}_{\mathrm{RD}}}\right] \\
& {\left[\delta \underline{x}_{D}^{-}+\sum_{k=1}^{p} \stackrel{*}{\Phi}_{D k} \stackrel{*}{M} \underline{c}_{k}\right]=0}
\end{aligned}
$$

There are $(m-1)$ independent relations of this type that must be satisfied. These are combined in a vector equation as follows:

$$
\delta \underline{\gamma}_{\mathrm{VTA}}=\stackrel{*}{\mathrm{~L}} \underline{\delta x}_{\mathrm{D}}{ }^{-}+\stackrel{*}{\mathrm{~L}} \sum_{\mathrm{k}=1}^{\mathrm{p}} \stackrel{*}{\Phi}{ }_{\mathrm{Dk}} \stackrel{*}{\mathrm{M}} \underline{\mathrm{c}}_{\mathrm{k}}=\underline{0}_{\mathrm{m}-1}
$$

where

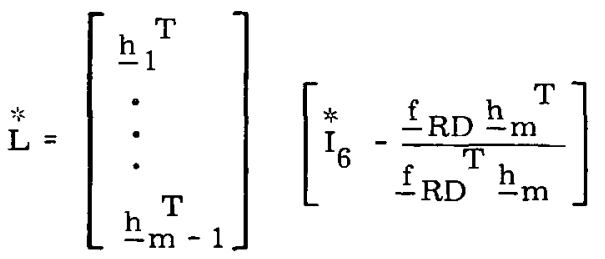

Equation (2.21) indicates that $m$ constraints at the variable end point $t_{A}$ in VTA guidance are equivalent to only $(m-1)$ constraints at the nominal end point $t_{D}$.

Constraint equations (2.17) and (2.21) may be generalized in the single equation

$$
\sum_{\mathrm{k}=1}^{\mathrm{p}} \stackrel{*}{\mathrm{P}}_{\mathrm{k}} \underline{\mathrm{c}}_{\mathrm{k}}+\underline{\rho}=\underline{0} \mathrm{n}
$$

where

$$
\begin{aligned}
& \left(\stackrel{*}{P_{k}}\right)_{\text {FTA }}=\stackrel{*}{H} \stackrel{*}{\Phi}_{D k} \stackrel{*}{M} \\
& \left(\stackrel{*}{P}_{k}\right)_{\text {VTA }}=\stackrel{*}{L^{*}}{ }_{D k}^{*} \stackrel{*}{M} \\
& \left(\underline{\rho}_{\text {FTA }}={ }^{H} \delta_{x_{D}}^{-}\right.
\end{aligned}
$$




$$
(\underline{p})_{\mathrm{VTA}}=\stackrel{*}{\mathrm{~L}} \delta \underline{x}_{\mathrm{D}}
$$

$n$ is the number of constraints at $t_{D}$. The $n-$ dimensional vector $\underline{\rho}$ is known as the miss vector

The preceding discussion applies to arbitrary constraints, six or less in number, at the time of arrival at the destination. In many guidance systems the only quantities constrained are the components of the vehicle's final position relative to the target. For such systems Eqs. (2.17) and (2. 21) can be simplified considerably, as shown below.

When final position relative to the target is completely specified and there are no other constraints, the basic vector equation of the constraints is

$$
\underline{\gamma}=\underline{r}_{\text {RA }}=\underline{0}_{3}
$$

For FTA guidance

$$
\begin{aligned}
& \delta \gamma_{\mathrm{FTA}}=\delta \underline{r}_{\mathrm{D}} \\
& \stackrel{*}{\mathrm{H}}=\left[\begin{array}{lll}
* & * & * \\
\mathrm{I}_{3} & 0_{3}
\end{array}\right] \\
& \left(\mathrm{*}_{\mathrm{k}}^{*}\right)_{\mathrm{FTA}}=\stackrel{\mathrm{*}}{\mathrm{*}}_{\mathrm{Dk}}
\end{aligned}
$$

so that the final guidance equations are given by

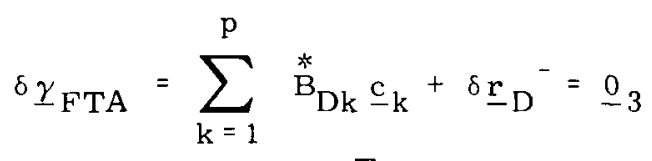

For VTA guidance, $\underline{h} \mathrm{~m}$ can be taken as

$$
\underline{h}_{\mathrm{m}}^{\mathrm{T}}=\left[\begin{array}{llllll}
0 & 0 & 1 & 0 & 0 & 0
\end{array}\right]
$$

Then

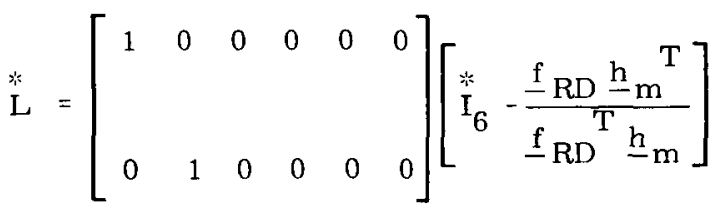

$$
\begin{aligned}
& =\left[\begin{array}{cccccc}
1 & 0 & -\frac{v_{R D x}}{v_{R D z}} & 0 & 0 & 0 \\
0 & 1 & -\frac{v_{R D y}}{v_{R D z}} & 0 & 0 & 0
\end{array}\right] \\
& \left(\mathrm{P}_{k}\right)_{\mathrm{VTA}}=\left[\begin{array}{ccc}
1 & 0 & -\frac{\mathrm{v}_{\mathrm{RDx}}}{\mathrm{v}_{\mathrm{RDz}}} \\
0 & 1 & -\frac{{ }^{\mathrm{RDy}}}{\mathrm{v}_{\mathrm{RDz}}}
\end{array}\right] \stackrel{\mathrm{B}}{\mathrm{Bk}}^{*}
\end{aligned}
$$

where $v_{R D x}, v_{R D y}, v_{R D z}$ are the components of the relative velocity vector $\mathrm{V} R D$. With these relations substituted into (2.21), the VTA guidance relations reduce to

$$
\delta \underline{\gamma}_{\text {VTA }}=\stackrel{*}{L}, \sum_{\mathrm{k}=1}^{\mathrm{p}} \stackrel{*}{\mathrm{~B}}_{\mathrm{Dk} \underline{\mathrm{c}}_{\mathrm{k}}}+\stackrel{*}{\mathrm{~L}}{ }^{\prime} \delta \underline{\mathrm{r}}_{\mathrm{D}}{ }^{-}=\underline{0}_{2}
$$

where $\mathrm{L}^{*}$ is the "reduced" $\mathrm{L}$ matrix.

$$
*^{\prime}=\left[\begin{array}{ccc}
1 & 0 & -\frac{{ }^{v_{R D x}}}{v_{R D z}} \\
0 & 1 & -\frac{v_{R D y}}{v_{R D z}}
\end{array}\right]
$$

The miss vector is simply $\delta \underline{\mathrm{r}^{-}}{ }^{-}$for positionconstrained FTA guidance while for positionconstrained VTA guidance it is the two-dimensional vector ( $L^{A} \delta_{\underline{r}}{ }^{-}$).

\section{CRITICAL-PLANE COORDINATE SYSTEM}

A special rotating coordinate system, called the critical-plane coordinate system, is developed in this section for the purpose of simplifying the analysis of VTA guidance. by

If $\mathrm{B}_{\mathrm{Dk}}$ is nonsingular, the vector $\mathrm{w}_{k}$ is defined

$$
\underline{\mathrm{w}}_{\mathrm{k}}=\stackrel{\text { * }}{\mathrm{B}}_{\mathrm{Dk}}^{-1} \underline{\mathrm{v}}_{\mathrm{RD}}
$$

Consider the effect of premultiplying $\underline{\mathrm{v}} \mathrm{RD}$ by $\stackrel{\mathrm{L}}{\mathrm{L}}^{\prime}$ and of premultiplying $\underline{w}_{k}$ by $\left(L^{r}{ }^{B} \mathrm{Dk}\right)$.

$$
\begin{aligned}
& \stackrel{*}{\mathrm{~L}}^{\prime} \underline{\mathrm{v}}_{\mathrm{RD}}=\underline{0}_{2}
\end{aligned}
$$

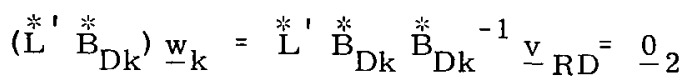

If the predicted position variation $\delta \mathrm{r} \mathrm{D}^{-}$is parallel to $\mathrm{v} R \mathrm{D}^{\prime} \mathrm{L}^{\prime} \delta \mathrm{r} \mathrm{D}^{-}$in Eq. $(2.30)^{-}$is equal to $\underline{0}_{2}$, and no corrections are required; thus the component of any given $\delta \mathrm{r} \mathrm{D}^{-}$that is parallel to $V R D$ has no effect on the VTA constraints. Similarly, the component of the velocity correction $\mathrm{c}_{\mathrm{k}}$ that is parallel to $\mathrm{w}_{\mathrm{k}}$ has no effect on the constraints. For every correction time $t_{k}$ there is some direction in which a small velocity step produces a position variation at $t_{D}$ that is parallel to $\underline{\mathrm{V} D}$ and hence has no effect on the constraints.

The physical interpretation of these observations is that the VTA constraints are satisfied if the vehicle's actual position at the nominal time of arrival is on the line through the nominal target point parallel to $\underline{\mathrm{v}} \mathrm{RD}$. Position-constrained 
FTA guidance is guidance to a specified point in position space at a specified time; positionconstrained VTA guidance is guidance to a specified line in position space at the same specified time. As indicated by Eq. (2.29),

$$
\left(\delta \underline{r}_{D}\right)_{\mathrm{FTA}}=\underline{0}_{3}
$$

while for VTA,

$$
\left(\delta \underline{\mathbf{r}}_{\mathrm{D}}\right)_{\mathrm{VTA}}=-\underline{\mathrm{v}}_{\mathrm{RD}} \delta \mathrm{t}_{\mathrm{D}}
$$

The direction of $\mathrm{v} R D$ is designated the noncritical direction at $t_{D}$, and the direction of $\mathrm{w}_{\mathrm{k}}$ is designated the noncritical direction at $t_{k}$. The noncritical direction varies with $t_{k}$ because the elements of BDk are functions of $t_{k}$. As $t_{k}$ approaches $t_{D}$. $B_{D k}$ approaches singularity; $w_{k}$ approaches infinity in magnitude, and its direction approaches that of $\underline{v} R D$. Thus, stipulating that $\mathrm{v}$ RD defines the noncritical direction at $t_{D}$ is consistent with stipulating that $w_{k}$ defines the noncritical direction at $t_{k}$. $w_{k}$ is the noncritical vector. The plane perpendicular to $\mathrm{w}$ is called the critical plane; only the components of velocity correction in this plane can affect the constraints.

The concept of the critical plane and the noncritical direction can be exploited by the formulation of a coordinate system in which two of the axes lie in the critical plane and the third axis is in the noncritical direction. This system is a rotating system due to the dependence of $\mathrm{w}_{\mathrm{k}}$ on $t_{k}$. It will be referred to as the criticalplane coordinate system. The subscript $W$ will be used to indicate that a vector or matrix is expressed in the critical-plane system.

Figure 1 illustrates the relation between the critical-plane system axes, labeled $\xi \eta \zeta$, and the axes $x \mathrm{y} z$ of the reference coordinate system. The $\xi$ and $\eta$ axes are in the critical plane, the $\xi$ axis lying along the line of intersection of the critical plane with the reference plane; the $\zeta$ axis is in the noncritical direction, and the $\eta$ axis is so directed that $\xi \eta \zeta$ form a righthanded orthogonal triad. Angles $\theta$ and $\phi$ serve to align $\xi \eta \zeta$ relative to $x \mathrm{y} z$. The range of $\theta$ is $0^{\circ}$ to $360^{\circ}$; the range of $\phi$ is $0^{\circ}$ to $180^{\circ}$.

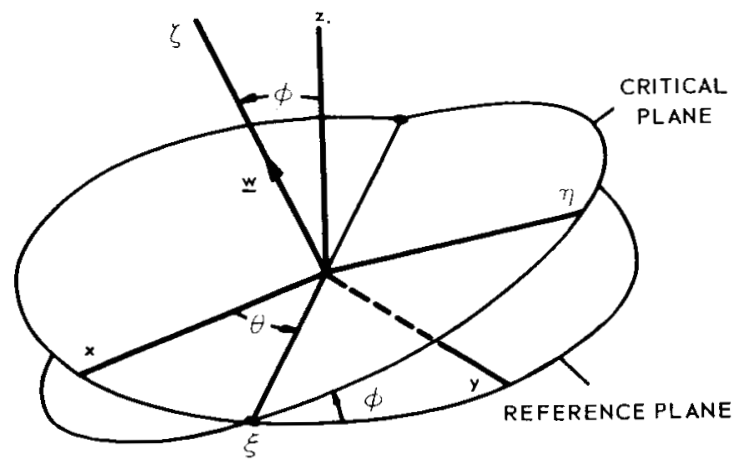

Fig. 1. Orientation of critical-plane coordinate system.
The vector $r$ is transformed from the ref erence coordinate system to the critical-plane coordinate system by the orthogonal transformation matrix $X$.

$$
\underline{r}_{W}=\left[\begin{array}{c}
\xi \\
\eta \\
\zeta
\end{array}\right]=\stackrel{* k}{x} \underline{\underline{x}}=\stackrel{*}{x}\left[\begin{array}{l}
x \\
y \\
z
\end{array}\right]
$$

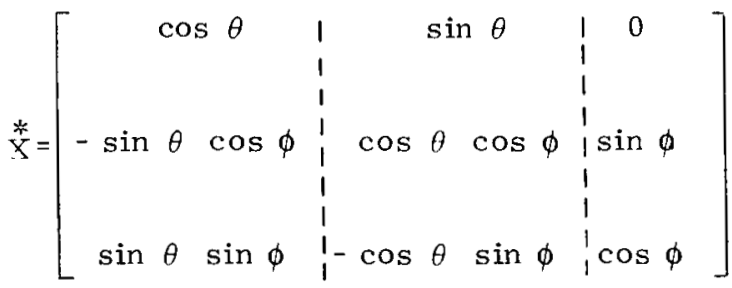

From the definition of an orthogonal matrix,

$$
\mathrm{x}^{-1}=\text { * }_{\mathrm{x}}^{\mathrm{T}}
$$

The transformations for $\underline{v}_{R D}$ and $\underline{w}_{k}$ are

$$
\begin{aligned}
\underline{v}_{R D} & =\left[\begin{array}{c}
v_{R D x} \\
v_{R D y} \\
v_{R D z}
\end{array}\right]={ }_{X_{D}^{*}}^{*}\left(\underline{v}_{R D}\right)_{W}=v_{R D}{ }^{*}{ }_{D}^{T}\left[\begin{array}{l}
0 \\
0 \\
1
\end{array}\right] \\
& =v_{R D}\left[\begin{array}{c}
\sin \theta_{D} \sin \phi_{D} \\
-\cos \theta_{D} \sin \phi_{D} \\
\cos \phi_{D}
\end{array}\right]
\end{aligned}
$$

$$
\underline{w}_{k}=\left[\begin{array}{c}
w_{k x} \\
w_{k y} \\
w_{k z}
\end{array}\right]=\stackrel{x}{k}_{k}{ }^{T}\left(\underline{w}_{k}\right)_{W}={ }^{*} \stackrel{*}{x}_{k}^{T}\left[\begin{array}{c}
0 \\
0 \\
1
\end{array}\right]
$$

$$
=w_{k}\left[\begin{array}{c}
\sin \theta_{k} \sin \phi_{k} \\
-\cos \theta_{k} \sin \phi_{k} \\
\cos \phi_{k}
\end{array}\right]
$$

$v_{R D}$ and $w_{k}$, without the underlining, are the magnitudes of $v R D$ and $w k$, respectively. From these equations the orientation angles can be found in terms of the components of ${ }_{R D}$ and $\underline{w}$ in the reference coordinate system. 


$$
\begin{aligned}
\cos \phi_{\mathrm{D}} & =\frac{{ }_{\mathrm{R}} \mathrm{Rz}}{\mathrm{v}_{\mathrm{RD}}} \\
\tan \theta_{\mathrm{D}} & =\frac{{ }^{\mathrm{r}} \mathrm{RD}}{-\mathrm{v}_{\mathrm{RDy}}} \\
\cos \phi_{\mathrm{k}} & =\frac{\mathrm{w}_{\mathrm{kz}}}{\mathrm{w}_{\mathrm{k}}} \\
\tan \theta_{\mathrm{k}} & =\frac{\mathrm{w}_{\mathrm{kx}}}{-\mathrm{w}_{\mathrm{ky}}}
\end{aligned}
$$

The vector relationship of Eq. (2.30) can be further simplified if it is expressed in the critical-plane coordinate system. The matrices involved are $\mathrm{L}^{\prime}$ and BDk. From (2.31),

$$
\left({ }^{*}{ }^{*}\right)_{W}=\left[\begin{array}{lll}
1 & 0 & 0 \\
0 & 1 & 0
\end{array}\right]
$$

$\left(\stackrel{*}{\mathrm{~B}}_{\mathrm{Dk}}\right) \mathrm{W}$ can be obtained from $\stackrel{*}{\mathrm{~B}}_{\mathrm{Dk}}$, the elements of which are computed by numerical integration, by a matrix transformation which can be derived from Eqs. (2.4), (2.5), and (2.6). If $\delta \underline{r}_{i}$ is zero and there are no velocity corrections be tween $t_{i}$ and $t_{j}$, then

$$
\begin{aligned}
& \delta \underline{r}_{j}=\stackrel{*}{*}_{j i} \underline{\delta}_{i} \\
& \stackrel{*}{X}_{j}^{T}\left(\delta \underline{r}_{j}\right)_{W}=\stackrel{*}{B}_{j i} \stackrel{*}{X}_{i}^{T}\left(\delta \underline{v}_{i}\right)_{W} \\
& \left(\delta \underline{r}_{j}\right)_{W}=\left(\stackrel{*}{X}_{j} \stackrel{*}{B}_{j i} \stackrel{*}{X}_{i}^{T}\right)\left(\delta \underline{v}_{i}\right)_{W}
\end{aligned}
$$

Thus,

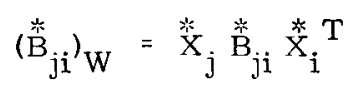

Correspondingly,

$$
\left(\stackrel{*}{*}_{D k}\right)_{W}=\stackrel{*}{x}_{D} \stackrel{*}{*}_{D k} \stackrel{*}{x}_{k}^{T}
$$

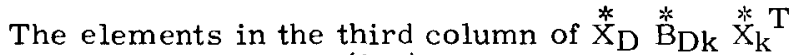
can be derived from (3.1).

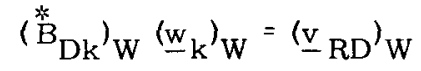

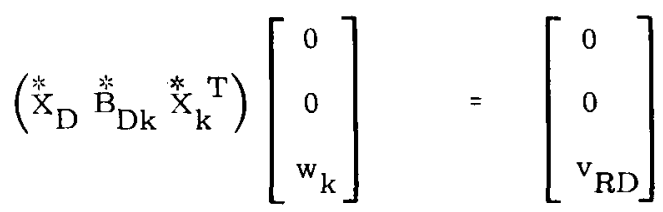

For this equation to be valid for non-zero $w_{k}$, it is necessary that the elements in the third column of the triple scalar product be zero, zero, and $\mathrm{v}_{\mathrm{RD}} / \mathrm{w}_{\mathrm{k}} \cdot$ Consequently,

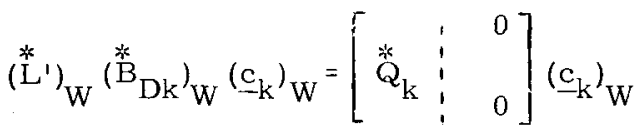

$$
\begin{aligned}
& =\hat{Q}_{k}^{*} a_{k}
\end{aligned}
$$

where $\stackrel{*}{Q}_{k}$ is the 2-by-2 matrix consisting of the elements in the first two rows and the first two columns of $\hat{X}_{D} B_{D k} \dot{X}_{k}{ }^{T}$, and $\underline{a}_{k}$ is the twodimensional vector consisting of the components of $c \mathrm{k}$ along the $\xi_{\mathrm{k}}$ and $\eta_{\mathrm{k}}$ axes in the critical plane. Then the vector equation of the constraints is

$$
\sum_{k=1}^{p} \stackrel{*}{*}_{k} a_{k}+\underline{\rho}=\underline{0}_{2}
$$

Here miss vector $\rho$ is the projection of of $\delta \underline{r}_{D}{ }^{-}$in the critical plane.

Eq. (3.24) brings out the fundamental advantage of the critical-plane concept. With this concept the constraints of the VTA guidance problem, which is physically a three-dimensional problem, can be expressed mathematically in a rotating two-dimensional coordinate system. The concept is exploited in the following sections in devising optimum midcourse correction strategies.

\section{SINGLE-CORRECTION STRATEGY}

If the number of constraints at $t_{D}$ is equal to or less than three, a single velocity correction normally is sufficient to satisfy the se constraints. In this section a method is developed for deter mining the time at which to apply a single correction such that the magnitude of the correction is a minimum.

In position-constrained FTA guidance, for which the number of constraints at $t_{D}$ is three, the single correction C FTA, applied at time $t_{C}$, that satisfies the constraints, is, according to Eq. (2.32),

$$
\underline{\mathrm{c}}_{\mathrm{FTA}}=-\stackrel{\mathrm{k}}{\mathrm{B}} \mathrm{DC}^{-1} \delta \underline{\mathrm{r}}_{\mathrm{D}}^{-}
$$

This equation can be solved as long as $t_{C}$ is such that $\mathrm{BDC}_{\mathrm{DC}}$ is not singular.

In position-constrained VTA guidance, for which there are only two constraints at $t_{D}$, the single correction C VTA satisfies the constraints if $a$, its projection in the critical plane corresponding to ${ } \mathrm{C}$, is given by

$$
\underline{a}=-\stackrel{k}{Q}_{C}^{-1} \underline{\rho}
$$

Time $t_{C}$ is chosen such that ${ }^{*}{ }_{C}$ is not singular. The third component of CVTA, the one in the noncritical direction at $t_{C}$, does not affect the VTA 
constraints; obviously the magnitude of $\mathrm{C}$ VTA is minimized if the third component is zero, so that

$$
\underline{c}_{\text {VTA }}=\left[\begin{array}{l}
\underline{a} \\
0
\end{array}\right]
$$

For either FTA or VTA the magnitude of the correction depends on $t_{C}$ and the corresponding miss vector. The object now is to determine the $t_{C}$ which minimizes the magnitude of $c$ when $\delta \underline{r} D$ or $\underline{p}$ is specified. Because it is simpler to analyze, the VTA case will be considered first.

Miss vector $\underline{\rho}$ can be expressed in targetcentered polar coordinates $\rho$ and $\psi$ in the critical plane. $\rho$ is the magnitude of the vector $\rho$; $\psi$ is the angle between $\underline{\rho}$ and the $\xi$ D axis.

$$
\begin{aligned}
& \underline{\rho}=\left[\begin{array}{c}
\delta \xi_{\mathrm{D}}^{-} \\
\delta \eta_{\mathrm{D}}^{-}
\end{array}\right]=\rho\left[\begin{array}{l}
\cos \psi \\
\sin \psi
\end{array}\right] \\
& \psi=\tan ^{-1}\left(\frac{\delta \eta_{\mathrm{D}}^{-}}{\delta \xi_{\mathrm{D}}^{-}}\right)
\end{aligned}
$$

The square of the magnitude of $\underline{\mathrm{c}}$ VTA is

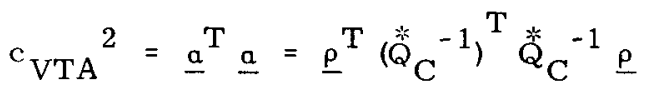

Then

$$
\begin{aligned}
c_{\text {VTA }}= & \rho\left\{\left[\begin{array}{ll}
\cos \psi & \sin \psi
\end{array}\right]\right. \\
& \left.\cdot\left({ }_{\mathrm{Q}}^{*}{ }^{-1}\right)^{\mathrm{T}} \stackrel{\mathrm{Q}}{\mathrm{Q}}^{-1}\left[\begin{array}{c}
\cos \psi \\
\sin \psi
\end{array}\right]\right\}^{1 / 2}
\end{aligned}
$$

The magnitude of $C$ VTA is a linear function of the magnitude of $\bar{\rho}$ but varies nonlinearly with $\psi$ and $t_{C}$. The only property of $\rho$ that affects the optimum time of correction is the angle $\psi$.

The optimum single-correction strategy is predetermined (before the flight) by computing and plotting a one-parameter family of curves, the parameter being $\psi$. The abscissa is the time of correction (or, equivalently, one of the anomalies), and the ordinate is the miss correctable per unit of velocity correction, i. e., the ratio of $\rho$ to $c_{V T A}$. For each value of $\psi$ there is generally a value of $t_{C}$ at which the miss correctable is a maximum; this is the optimum $t_{C}$ for that $\psi$. A cross-plot of optimum
${ }^{t} \mathrm{C}$ versus $\psi$ is the basis for selecting the time of correction to be used when some $\rho$ has been inferred from measurements made during the flight. For FTA guidance, $\delta \mathrm{r} \mathrm{D}^{-}$is expressed in terms of target-centered spherical coordinates. The magnitude of $c$ FTA varies linearly with the magnitude of $\delta \underline{\mathbf{r}} \mathrm{D}^{-}$but nonlinearly with the two angle coordinates. The optimum single correction strategy involves a two-parameter family of curves, the parameters being the two angles. Thus, the procedure is considerably more laborious than the one-parameter procedure outlined for VTA. However, the basic strategy is the same; curves of miss correctable versus ${ }^{t} \mathrm{C}$ are plotted for fixed values of the two angles, and the values of $t_{C}$ corresponding to the maxima of the individual curves are cross-plotted as functions of the two angles to indicate the optimum correction time for any given $\delta \mathrm{r}^{-}$.

The procedure is illustrated for VTA guidance in Figs. 2 and 3. The reference trajectory is an outbound (perifocus to apofocus) Hohmann transfer with eccentricity of 0.95 . The trajectory of the target is assumed to lie in the plane of the reference trajectory, and the relative velocity of the vehicle with respect to the target at $t_{D}$ is assumed to be parallel to the velocity of the vehicle relative to the attractive focus. Obviously, this is a highly simplified case, the reference trajectory being both two-dimensional and two-body; nevertheless the guidance problem is still threedimensional, because out-of-plane and in-plane components of the miss vector are both taken into consideration. The simplicity of the case reduces the amount of numerical computation, yet adequately illustrates the fundamental principles of single-correction strategy.

Physically in this trajectory the noncritical direction at $t_{D}$ is parallel to the minor axis of the reference ellipse. The critical plane at $t_{D}$ is perpendicular to the reference trajectory plane, and the line of nodes between the two planes is the major axis of the reference ellipse. Thus, when $\psi=0^{\circ}, \underline{p}$ is parallel to the major axis; when $\psi=90^{\circ}, \underline{\rho}$ is perpendicular to the reference plane.

The range of $\psi$ is $0^{\circ}$ to $360^{\circ}$. Since an increase of $180^{\circ}$ in $\psi$ between one miss vector and another changes the sign of the required correction but not its magnitude, the miss correctable, which is a ratio of magnitudes, is not affected, and hence the computations can, in general, be confined to the range $\psi=0^{\circ}$ to $\psi=180^{\circ}$. In this simple special case, there is also symmetry about $\psi=90^{\circ}$; that is, the miss correctable at $\left(90^{\circ}+\psi\right)$ is equal to the miss correctable at $\left(90^{\circ}-\psi\right)$ for the same $t_{C}$, so that the actual computations cover only the range $0^{\circ}$ to $90^{\circ}$ in $\psi$.

Fig. 2 is a plot of normalized miss correctable versus $E_{C}$, the eccentric anomaly at the time of correction. The range of $\mathrm{E}_{\mathrm{C}}$ for an outbound Hohmann transfer is $0^{\circ}$ to $180^{\circ}$. The normalized miss correctable is the miss correctable at the given $\psi$ and $\mathrm{E}_{\mathrm{C}}$ divided by the miss correctable at $\psi=0^{\circ}, \mathrm{E}_{\mathrm{C}}=0^{\circ}$. Curves are drawn for $\psi=0^{\circ}, 5^{\circ}, 15^{\circ}, 30^{\circ}, 60^{\circ}$, and $90^{\circ}$. The dotted curve in the figure is the locus 


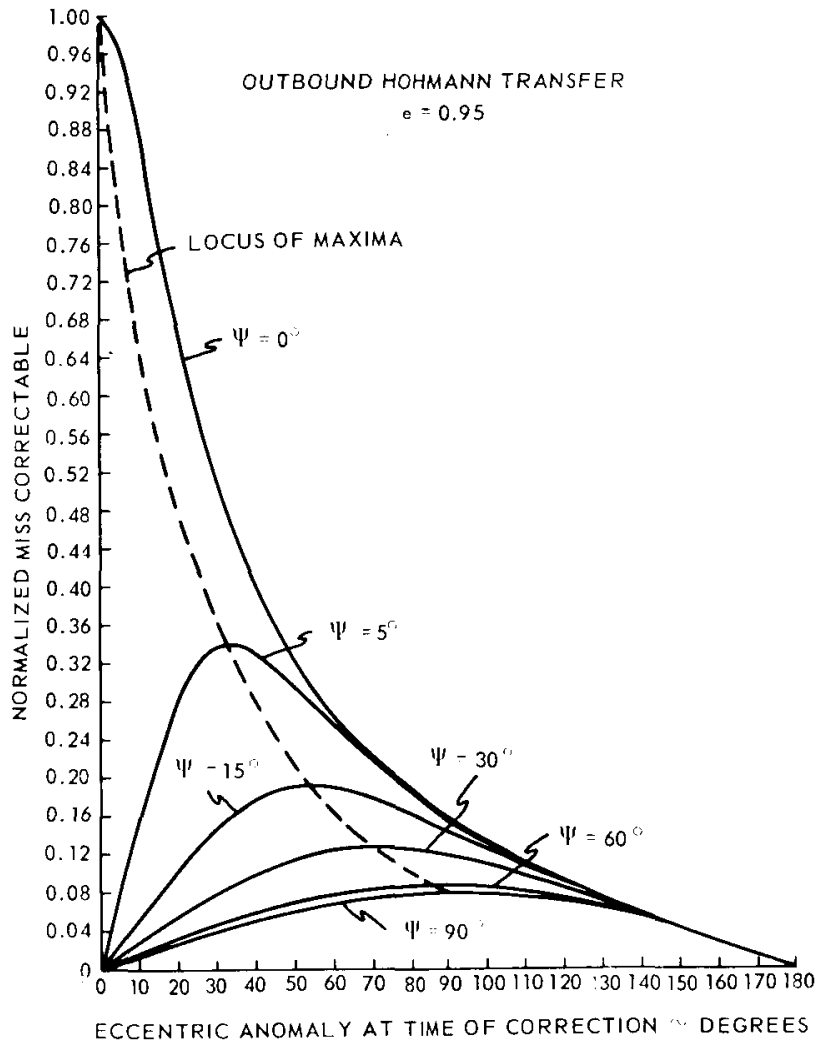

Fig. 2. Normalized miss correctoble versus eccentric anomaly of time of correction.

of the points of maximum miss correctable for $\operatorname{each} \psi$.

Fig. 3 is a re-plot of the dotted curve of Fig. 2. Eccentric anomaly at optimum correction time and maximum normalized miss correctable are plotted as functions of $\psi$. The optimum correction time curve is the only one that is needed to select the time of correction in an optimum singlecorrection VTA strategy

\section{MULTIPLE-CORRECTION STRATEGIES}

Section 4 presents a method of selecting the optimum time for a single midcourse correction which completely nullifies the miss vector at the destination. However, no consicleration has yet been given to the problem of multiple velocity corrections, i.e., of determining whether several partial corrections, made at different times, may result in a total magnitude of velocity change that is smaller than the magnitucte of the single correction already described.

It was shown above that the miss at the nominal time of arrival at the destination can be described by an $n$-dimensional vector where $n$ is the number of terminal constraints for FTA guidance and one less than the number of terminal constraints for VTA guidance. In this section, it will be shown that for a given set of injection errors there is an optimum volocity correction
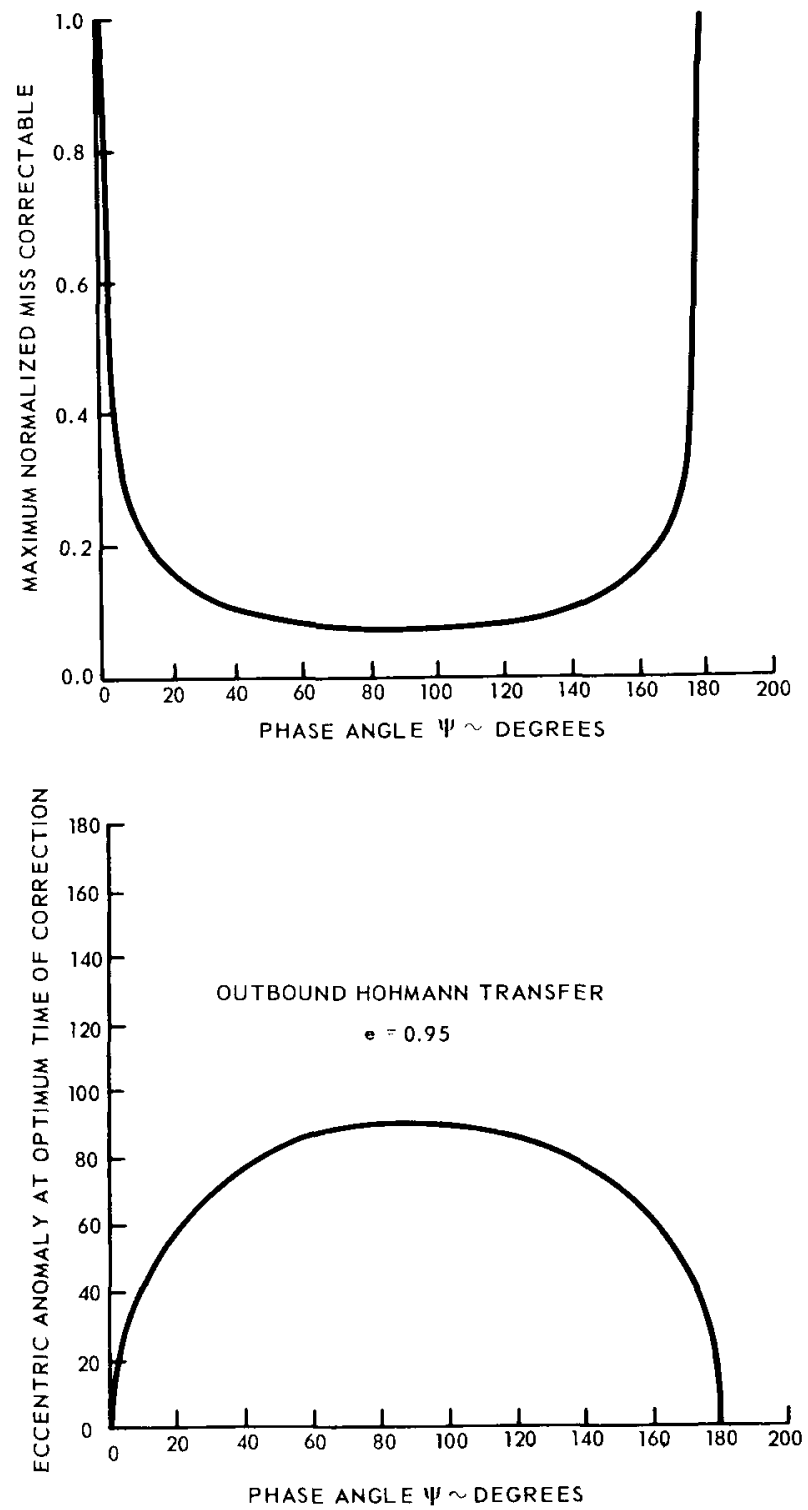

Fig 3. Maximum miss correctable and optimum eccentric anomaly at time of correction versus phase angle $\Psi$.

schedule consisting of at most $n$ velocity corrections which completely nulls out the miss due to injection errors.

The following relation between injection errors and velocity corrections was obtained in Section 2:

$$
\sum_{k=1}^{p} \stackrel{k}{P}_{k} \underline{c}_{k}+\underline{\rho}=\underline{0}_{n}
$$

where $\rho$ is the $\mathrm{n}$ dimensional vector representing the miss that would occur without velocity corrections, $c k$ represents the $k$ th velocity correction applied at time $t_{k}$ and $\hat{P}_{k}$ is the influence matrix relating the $k$ th velocity correction to the miss. 
It will be shown below that any velocity correction schedule containing more than $n$ corrections can be reduced to an $\mathbf{n}$ correction schedule without increasing the total velocity change required. This result was obtained previously by Neustadt( $(7)$ It then remains to find the best correction schedule from among all possible schedules containing $\mathbf{n}$ or fewer corrections. This is always possible in principle, and a fairly simple graphical method for finding the optimum schedule in the case when $n$ is two will be described in the next section.

In reducing the $\mathrm{p}$ correction schedule of Eq. (5. 1) to an $n$ correction schedule, only $(n+1)$ corrections will be considered at a time. Therefore, let $\underline{\rho}$ denote the effect of the first $(n+1)$ velocity corrections on the miss.

$$
\underline{\rho}_{a}=-\sum_{k=1}^{n+1}{\stackrel{*}{P_{k}}}_{k} \underline{c}_{k}
$$

The effect of the $(n+1)$ corrections is to reduce the miss from $\underline{\rho}$ to $(\underline{\rho}-\underline{\rho})$.

It will be shown that there is an $n$ correction schedule which uses no more total velocity change (probably less) than the $(n+1)$ corrections of Eq. (5.2) and results in the same corrective effect $\rho_{a}$. This reduces the summation in Eq. (5.1) to $7 \mathrm{p}-1)$ terms. Applying this reduction $(p-n)$ times results in an $n$ correction schedule which is at least as economical as the original $\mathrm{p}$ correction schedule.

To carry out the reduction of the Eq. (5-2) schedule to $\mathrm{n}$ corrections, let

$$
\underline{\mathrm{u}}_{\mathrm{k}}=\frac{1}{\mathrm{c}_{\mathrm{k}}} \stackrel{\text { *ै }}{\mathrm{P}}_{\mathrm{k}} \underline{\mathrm{c}}_{\mathrm{k}}
$$

Then

$$
\underline{\rho}_{a}=-\sum_{k=1}^{n+1} c_{k} \underline{u}_{k}
$$

Since $\underline{u}_{k}$ is an $n$-dimensional vector, the vectors $u_{1}, \ldots, \underline{u}_{n}+1$ are linearly dependent, and there are scalars $\lambda_{1}, \ldots, \lambda_{n}+1$ such that

$$
\sum_{k=1}^{n+1} \lambda_{k} \underline{u}_{k}=\underline{0}_{n}
$$

Let

$$
\lambda=\sum_{\mathrm{k}=1}^{\mathrm{n}+1} \lambda_{\mathrm{k}}
$$

If $\lambda$ is negative, the signs of all the $\lambda_{k}$ 's are changed so that

$$
\lambda \geq 0
$$

Now, let

$$
\mathrm{a}_{\mathrm{k}}=\frac{\lambda_{\mathrm{k}}}{\mathrm{c}_{\mathrm{k}}}
$$

and choose $r$ so that $a_{r}$ is the maximum of the $a_{k}{ }^{\prime} s$. Then $a_{r} \geq a_{k}$ for $k=1, \ldots, n+1$ and $a_{r}>0$ since $\lambda \geq 0$. Multiplying Eq. (5.5) by $1 / \mathrm{a}_{\mathrm{r}}$ and adding the result to Eq. (5.4) yields

$$
\underline{\rho}_{\mathbf{a}}=-\sum_{\mathrm{k}=1}^{\mathrm{n}+1} \mu_{\mathrm{k} \underline{u}_{\mathrm{k}}}
$$

with

$$
\mu_{k}=c_{k}-\frac{\lambda_{k}}{a_{r}}=\frac{c_{k}}{a_{r}}\left\{a_{r}-a_{k}\right\}
$$

By the last equality above $\mu_{k} \geq 0$ and $\mu_{r}=0$. Finally, by Eqs. (5. 3) and (5.9) it follows that

$$
\underline{\rho}_{a}=-\sum_{k=1}^{n+1} \stackrel{*}{*}_{k} c_{k}{ }_{k}^{\prime}
$$

with

$$
\underline{c}_{k}^{\prime}=\frac{\mu_{k}}{c_{k}} \underline{c}_{k}
$$

Eq. (5. 11) represents an $n$ correction schedule since $c r$ is zero. The total velocity change required by the new correction schedule is

$$
\begin{aligned}
c^{\prime} & =\sum_{k=1}^{n+1} c_{k}^{\prime}=\sum_{k=1}^{n+1} \mu_{k} \\
& =\sum_{k=1}^{n+1} c_{k}-\frac{\lambda}{a_{r}}
\end{aligned}
$$

Since $\lambda \geq 0, c^{\prime}$ is less than or equal to $c_{1}+\ldots$ $+\mathrm{c}_{\mathrm{n}}+\overline{1}$, and thus the new correction schedule is at least as economical as the old schedule. 
6. MULTIPLE-CORRECTION STRATEGY FOR POSITION-CONSTRAINED VTA GUIDANCE

The multiple correction case for positionconstrained VTA guidance is studied in this section. It was shown in the preceding section that the optimum velocity correction schedule for position-constrained VTA guidance need never contain more than two corrections (in some cases it consists of only one correction). This section outlines a geometric construction for determining the optimum set of velocity corrections needed to null out the miss due to given injection errors.

The analysis in this section makes extensive use of the equation of a line segment joining two given vectors in the target critical plane. Thus, in Fig. 4, the vectors $\mathrm{a}$ and $\underline{b}$ define a line segment $\overline{\mathrm{AB}}$. If the head of the vector $\mathrm{h}$ lies on this line segment and $\lambda$ denotes the ratio of $\overline{\mathrm{HB}}$ to $\bar{A} \bar{B}$, then

$$
\underline{\mathrm{h}}=\underline{\mathrm{b}}+\lambda(\underline{\mathrm{a}}-\mathrm{b})=\lambda \underline{\mathrm{a}}+(1-\lambda) \underline{\mathrm{b}}
$$

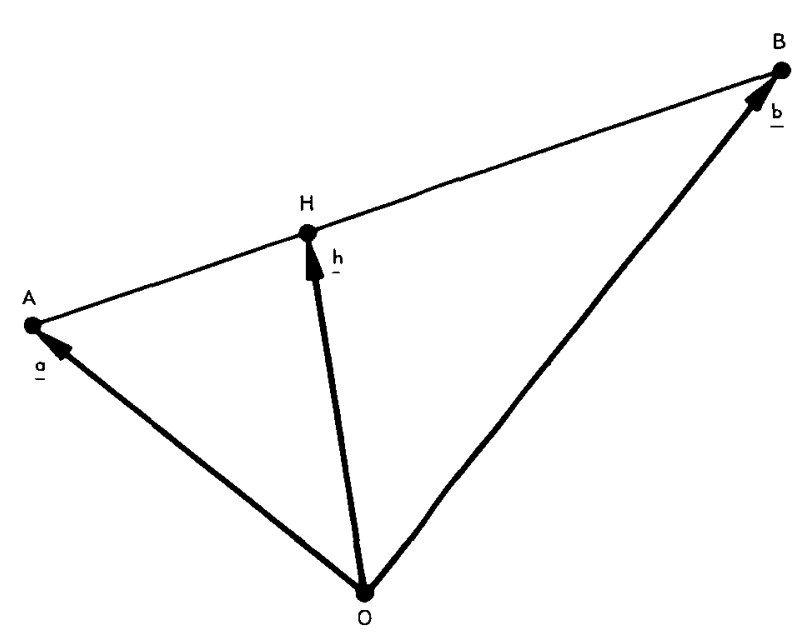

Fig. 4. Vector relation in the critical plane.

As the point $H$ moves a long the line segment, $\lambda$ ranges from zeroto one. Thus the equation of the line segment $\overline{\mathrm{AB}}$ is

$$
\underline{\mathrm{h}}=\lambda \underline{\mathrm{a}}+(1-\lambda) \underline{\mathrm{b}}, 0 \leq \lambda \leq 1
$$

Suppose that the miss vector $\rho$ due to injection errors is given and it is desired to find the optimum velocity correction schedule which nulls out this miss. Since the use of two corrections may result in a saving in total velocity change, the total corrective effect will be written as the sum of two components $\underline{\rho}_{1}$ and $\underline{\rho}_{2}$, to be produced by separate velocity corrections Thus, with

$$
\underline{\rho}_{\mathrm{k}}=\stackrel{*}{\mathrm{P}}_{\mathrm{k} \underline{\mathrm{c}}}=\mathrm{c}_{\mathrm{k}} \underline{\mathrm{u}}_{\mathrm{k}} ; \mathrm{k}=1,2
$$

Eq. (5. 1) becomes

$$
\underline{\rho_{1}}+\underline{\rho_{2}}=-\underline{p}
$$

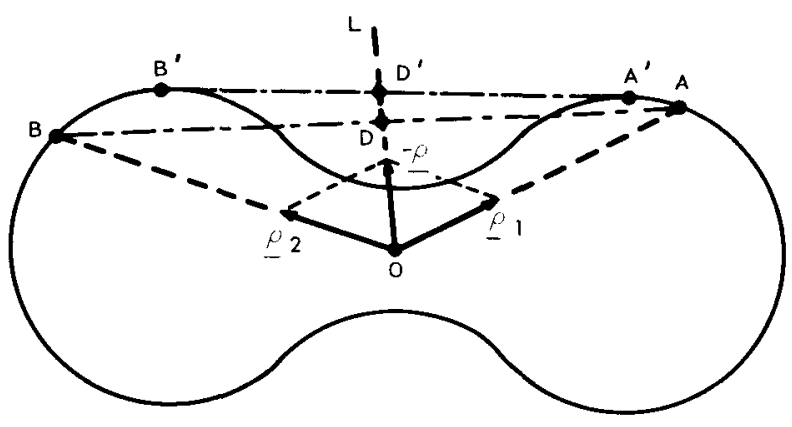

Fig. 5. Construction for optimum double velocity correction.

Fig. 5 illustrates this vector sum. The solid curve in the figure is a polar plot of maximum miss correctable as a function of $\psi$; that is, it represents the same type of information as that shown in the upper half of Fig. 3

In order to obtain the minimum total velocity change, corrective effects $\underline{\rho}_{1}$ and $\underline{\rho}_{2}$ should each be obtained by use of the single-correction strategy of Section 4 ; i. e., the correction which produces $\rho_{k}$ is made at the time for which the miss correctable is maximum for the $\psi$ corresponding to $\rho_{\mathrm{k}}$. If $A$ and $B$ are the points where lines drawn from the origin along vectors $\rho_{1}$ and $\rho_{2}$ intersect the solid curve, then, since the solid curve is the locus of the maximum corrective effect of a unit velocity change and since $\underline{u}_{k}$ is defined in Eq. (5.3) as the corrective effect of a unit velocity change, $\underline{u}_{1}$ and $\underline{u}_{2}$ are the vectors from the origin to $A$ and $B$, respectively. The magnitude of the correction that produces $\rho_{k}$ is simply the ratio of the magnitude of $\rho_{k}$ to the magnitude of $\underline{u}_{k}$.

$$
c_{k}=\frac{\rho_{k}}{u_{k}} \quad ; \quad k=1,2
$$

Fig. 5 illustrates this ratio; the equation follows directly from Eq. (6.3). The sum of the magnitudes of the two constituents of the multiple correction is

$$
c_{12}=c_{1}+c_{2}
$$

Since $\rho$ is given, minimizing $c_{12}$ is the same as maximizing the ratio $\rho / c_{12}$. This ratio represents the miss correctable with unit total velocity change when the required corrective effect $-\underline{p}$ is divided into components parallel to $\underline{\rho}_{1}$ and $\underline{\rho}_{2}$.

$$
\begin{aligned}
\frac{-\underline{p}}{\bar{c}_{12}} & =\frac{\underline{\rho} 1+\underline{p}}{c_{12}} \\
& =\lambda \underline{u}_{1}+(1-\lambda) \underline{u}_{2}
\end{aligned}
$$

where

$$
\lambda=\frac{\mathrm{c}_{1}}{\mathrm{c}_{1}+\mathrm{c}_{2}}
$$


Thus $0<\lambda<1$ and the head of the vector $-\rho / c_{12}$ must lie on the line segment $\overline{A B}$. However-p/c12 also lies on the line $L$ drawn from the origin along the vector- $p$, and therefore $p / c_{1}$ must be the vector from the origin to the point $D$ at the intersection of $\overline{\mathrm{AB}}$ and the line $\mathrm{L}$. Then $\rho / \mathrm{c}_{12}$ is the length of the line segment $\overline{\mathrm{OD}}$. More generally, this argument shows that the line segment $\bar{A} \bar{B}$ is the locus of miss vectors which can be corrected with unit total velocity change after being split up into components parallel to $\underline{u}_{1}$ and u 2 .

To maximize the ratio $\rho / c 12$, the points $A$ and $B$ must be moved around the solid curve until the intersection of $\overline{\mathrm{AB}}$ and $\mathrm{L}$ is farthest from the origin. This occurs when $A$ and $B$ are positioned so that the line segment $\overline{\mathrm{AB}}$ meets the solid curve at points of tangency, as the line segment $\bar{A}^{\prime} B^{\prime}$ does in Fig. 5.

If the line $L$ intersects the solid curve at a point where the solid curve is convex as in Fig. 6 and one tries to represent $-p$ as the sum of two partial corrections, one finds that the intersection of the line segment $\overline{\mathrm{AB}}$ with $\mathrm{L}$ is always closer to the origin than the intersection of $L$ with the solid curve and no double correction is as economical as the best single correction.

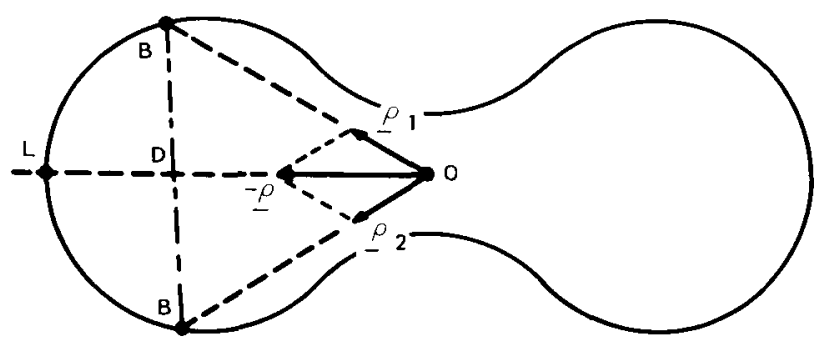

Fig. 6. Example of a vector $-\rho$ for which a single correction is optimum.

Finally, the locus $S$ of vectors $-\rho$ which are obtained with an optimum single or double velocity correction with unit total velocity change is made up of the convex parts of the curve for optimum single corrections plus the tangent line segments as illustrated in Fig. 7.

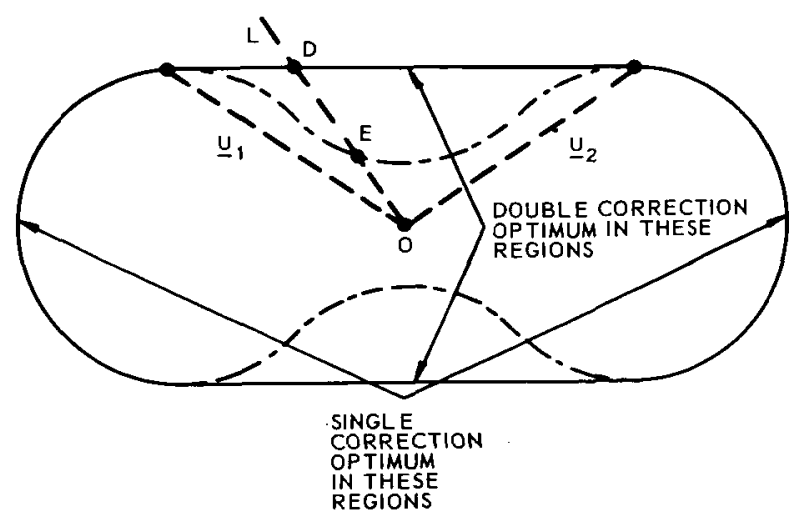

Fig. 7. Plot of corrective effect obtained with optimum unit double or single velocity correction.
The curve $S$ is the basis for calculating optimum velocity correction schedules. Suppose that it is desired to find the optimum correction schedule which produces the corrective effect - $\underline{p}$.

If the line $\mathrm{L}$ from the origin along the vector - $\rho$ intersects the curve S along a curved portion, - $\rho$ should be obtained by means of the best single correction. However if the line $L$ from the origin along the vector- $\rho$ intersects the curve $S$ along a straight line portion, $-p$ should be split up into components parallel to the vectors $u_{1}$ and $u_{2}$ from the origin to the ends of the straight line portion. This operation involves solving a pair of simultaneous linear equations. Thus, scalars a and $\beta$ must be found such that

$$
-\underline{\rho}=a \underline{u}_{1}+\beta \underline{u}_{2}
$$

where - $\underline{\rho}, \underline{u}_{1}$ and $\underline{u}_{2}$ are given. Writing this vector equation out in components results in two scalar equations which may be solved for $a$ and $\beta$ provided $\underline{u}_{1}$ and $\underline{u}_{2}$ are not collinear. $\underline{u}_{1}$ and $\underline{u}_{2}$ cannot be collinear since they extend from the origin to opposite ends of a line segment. Once $a$ and $\beta$ have been found, the optimum single correction technique of Section 4 may be applied to the vectors $a u_{1}$ and $\beta u_{2}$. In this case the ratio of the total velocity change required for the optimum double correction to that required for the optimum single correction is the ratio of the lengths of the line segments $\overline{\mathrm{OE}}$ and $\overline{\mathrm{OD}}$ in Fig. 7.

Figs. 8 and 9 are polar plots of the corrective effect produced by the best single velocity correction as a function of direction in the target critical plane for Hohmann transfers of various eccentricities. The components of corrective effect in the trajectory plane and perpendicular to the trajectory plane were plotted to different scales in order to make the curves more symmetrical. For the outbound transfers illustrated in Fig. 8, the curves become more concave as the eccentricity increases. For the 0.95 eccentricity outbound transfer, the curve indicates that, in the directions which favor a double correction the most, the best single correction uses about fifty percent more total velocity change than the best double correction. The curves for inbound Hohmann transfers in Fig. 9 are always convex, indicating that single corrections are always better than double corrections on inbound transfers of this type.

In more precise terms the locus of - $\rho$ vectors which are obtained with an optimum single or double velocity correction with total velocity change less than or equal to unity is the convex hull of the set of - $p$ vectors which may be obtained with optimum single corrections with total velocity change less than or equal to unity. A convex set is a set having the property that any line segment joining two points in the set lies entirely within the set. The convex hull of a set is the smallest convex set containing the original set. The operation of drawing in tangent line segments described above thus corresponds to constructing the convex hull of the set of $-\rho$ vectors which may be obtained with optimum single corrections with total velocity change less than or equal to unity. 


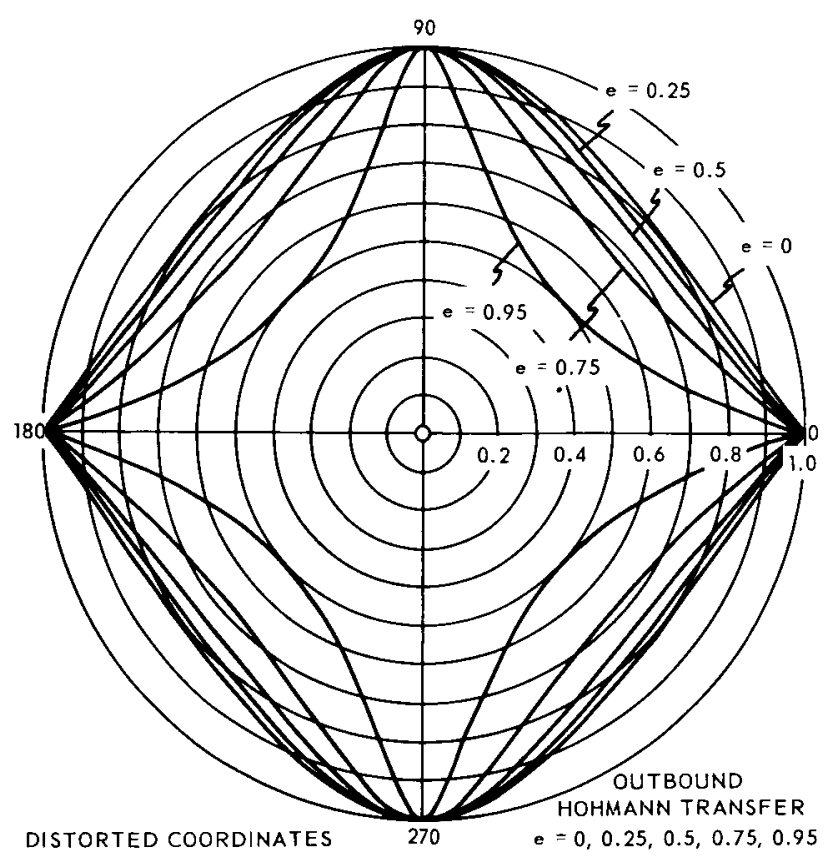

Fig. 8. Polar plot of normalized miss correctable versus phase angle $\Psi$.

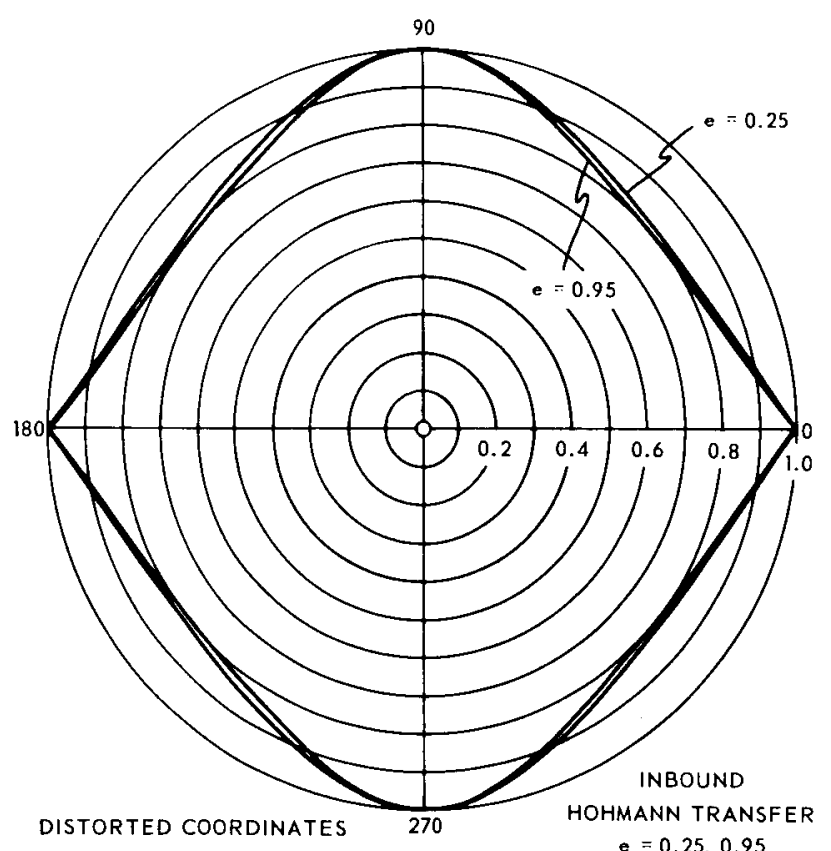

Fig. 9. Polar plot of normalized miss correctable versus phase angle $\Psi$.

NOMENCLATURE

General

An asterisk over a capital letter indicates a matrix.

An underlined lower-case letter indicates a column vector. A vector symbol without the underlining indicates the magnitude of the vector.
A single dot over a vector symbol indicates the first derivative with respect to time in an inertially non-rotating coordinate system.

Square brackets a round a partial derivative involving vector quantities indicates a column vector or matrix consisting of the partial deriv atives of the components of the vectors.

\section{English Symbols}

${ }^{a}$ ratio of $\lambda_{k}$ to the magnitude of $\underline{c}_{k}$

$a_{r} \quad$ maximum of the $a_{k}$ 's

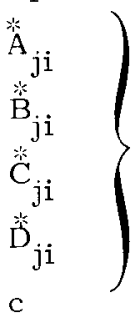

submatrices of the state transition matrix

single velocity correction which satisfies the const 1 ints

$\mathrm{k}$-th of a series of velocity corrections which, taken together, satisfy the constraints

sum of magnitudes of ${ }_{c}{ }_{k}$ 'vectors

$\mathrm{k}$-th velocity correction in revised schedule

sum of magnitudes of velocity corrections in a two-correction schedule.

rate of change of the state vector with time in the absence of any control action

FTA fixed-time-of-arrival six-dimensional vector of the partial de rivatives of constraint $\gamma_{k}$ with respect to the components of $\delta \underline{x}$.

m-by-6 matrix relating the constraints to $\delta \underline{x}_{D}$ in FTA guidance

$\mathrm{k}$-by-k identity matrix

(m - 1)-by-6 matrix relating the constraints to $\delta \underline{x}_{D}$ in VTA guidance 2-by-3 matrix relating position constraints to $\delta \underline{r}_{D}$ in VTA guidance

number of contraints at actual time of arrival

6 -by-3 compatibility matrix

number of constraints at nominal time of arrival

$\mathrm{k}$-component zero vector 
number of velocity corrections

$\stackrel{*}{\mathrm{P}}_{\mathrm{k}}$

$\stackrel{*}{\mathrm{Q}}_{\mathrm{k}}$

$\underline{r}$

S

t

$\underline{\mathrm{u}} \mathrm{k}$

$\underline{\mathrm{v}}$

$\mathrm{v}_{\mathrm{RD}}$

VTA

$\underline{\mathrm{w}}_{\mathrm{k}}$

$\mathrm{w}_{\mathrm{k}}$

$\underline{\mathbf{x}}$

X

transformation matrix from reference coordinate system to criticalplane coordinate system

axes of reference coordinate sys tem

z

Greek Symbols

a $\mathrm{k}$

$a$

$\beta$

$\gamma_{\mathrm{k}}$

$\gamma$

$\delta$

$\delta t_{D}$

$\delta\left(t-t_{k}\right)$

$\lambda$ component of velocity correction in the critical plane weighting factor relating $\underline{u}_{1}$ to $-\underline{\rho}$ weighting factor relating $\underline{u}_{2}$ to $-\underline{p}$

$\mathrm{k}$-th constraint

constraint vector

first variation

change in time of arrival at des tination

Dirac delta function at time $t_{k}$ sum of $(n+1) \lambda_{k}{ }^{\prime} s$ $\lambda$

$\lambda_{\mathrm{k}}$

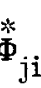

$\boldsymbol{\Phi}_{\mathrm{ji}}$

$\mu_{\mathrm{k}}$

$\underline{p}$

$\underline{p} \mathbf{a}$

$\psi$

\section{$\xi$}

$\eta$

$\zeta$

Subsc ripts

A

C

D

FTA

I

i

$\mathrm{k}$

k

R

$\mathrm{T}$

VTA

$\mathrm{x}$

y scalar quantity limited to the range zero to one

scalar coefficient associated with

$\underline{\mathrm{u}}_{\mathrm{k}}$

6-by-6 state transition matrix

weighting factor relating $\underline{u}_{k}$ to $\underline{\rho}_{a}$

miss vector

corrective effect of first $(n+1)$

velocity corrections

angle between miss vector and $\xi_{\mathrm{D}}$ axis

coordinate axes in the critical plane

coordinate axis in the noncritical direction

pertaining to actual time of arrival at destination

pertaining to time of a single velocity correction

pertaining to nominal time of arrival at destination

pertaining to fixed-time-of-arrival guidance

pertaining to nominal time of in jection

pertaining to time $t_{i}$

pertaining to time $\mathbf{t}_{j}$

pertaining to time $t_{k}$

dummy index

pertaining to the vehicle's condition relative to the target

pertaining to the target

pertaining to variable-time-ofarrival guidance

pertaining to critical-plane coordinate system

pertaining to vector components along the axes of the reference coordinate system 
$\left.\begin{array}{l}\xi \\ \eta \\ \zeta\end{array}\right\}$

pertaining to vector components along the axes of the critical-plane coordinate system

Superscripts

$\mathrm{T}$

transpose of a vector or matrix

$-$

$-1$

pertaining to conditions that would exist if no corrections were applied

inverse of a matrix

\section{ACKNOW LEDGEMENT}

The authors wish to express their appreciation to the United States National Aeronautics and Space Agency, which supported the preparation and presentation of this report under NASA Research Grant NsG 254-62. They also wish to thank Mrs. Linda P. Abrahamson and Mr. Berl P. Winston, who programmed the computations and prepared the figures used in the numerical example.

Acknowledgment is made of the MIT Computation Center for the work done on its computer under Problem Number V3008.

The publication of this report does not constitute approval by the National Aeronautics and Space Administration of the findings or the conclusions contained the rein. It is published only for the exchange and stimulation of ideas.

\section{REFERENCES}

(1) Battin, R. H. , Astronautical Guidance, McGraw-Hill, Inc., New York, 1964.

(2) Noton, A. R. M., Cutting, E., and Barnes, F. L., "Analysis of Radio-Command MidCourse Guidance," JPL Technical Report No. 32-28, Sept. 1960 .

(3) McLean, J. D., Schmidt, S. F., and McGee, L. A., "Optimal Filtering and Linear Prediction Applied to a Midcourse Navigation System for the Circumlunar Mission" NASA Technical Note D-1208, 1962.

(4) Breakwell, J. V., "Fuel Requirements for Crude Interplanetary Guidance," Advances in the Astronautical Sciences, Vol. 5 , Plenum Press, Inc., New York, 1960, pp. $53-65$.

(5) Breakwell, J. V., "The Spacing of Corrective Thrusts in Interplanetary Navigation," Advances in the Astronautical Sciences, Vol. 7, Plenum Press, Inc, , New York, 1961 , pp. $219-235$.

(6) White, J.S., Callas, G. P., and Cicolani, L.S., "Application of Statistical Filter Theory to the Interplanetary Navigation and Guidance Problem," NASA TN D-2697, 1965.

(7) Neustadt, L. W., "Optimization, a Moment Problen, and Nonlinear Programming," Journal of the Society for Industrial and Applied Mil hematics, Series A, Control, Vol. 2, voo. 1, Philadelptia, 1, 64, p. 33-53. 